\title{
Influencia del estado nutricional sobre el equilibrio postural en niños: un estudio piloto
}

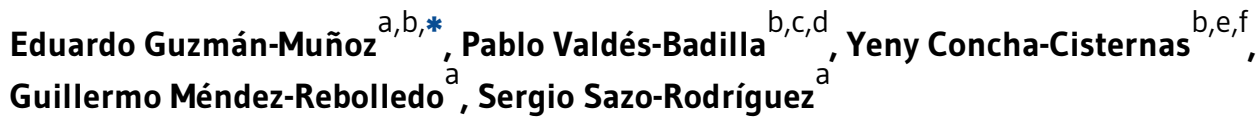

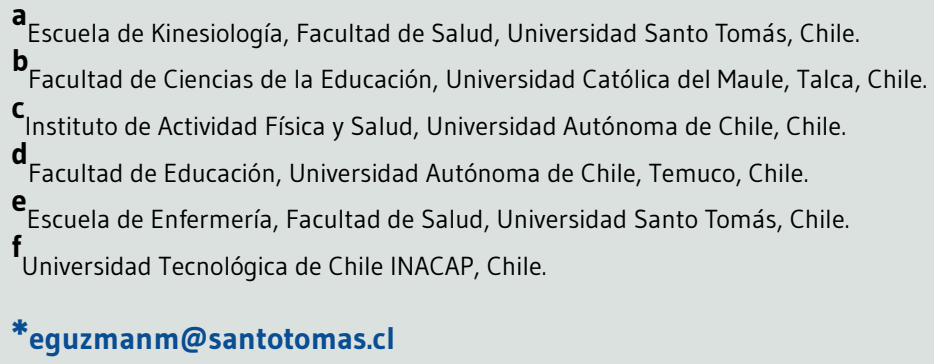

Recibido el 11 de octubre de 2016; aceptado el 23 de enero de 2017.

Influencia del estado nutricional sobre el equilibrio postural en niños: un estudio piloto

\section{PALABRAS CLAVE}

Sobrepeso;

Obesidad;

Equilibrio Postural;

Estado Nutricional;

Niños.

\section{RESUMEN}

Introducción: El aumento de masa corporal puede afectar la realización de tareas funcionales como el equilibrio y propiciar el riesgo de caídas y lesiones, sin embargo las pruebas en niños son limitadas. El propósito de este estudio fue determinar la influencia del estado nutricional sobre el equilibrio postural en niños entre 6 y 9 años de edad.

Material y Métodos: Estudio piloto descriptivo transversal. Se evaluaron 71 niños en cuanto a peso, talla y equilibrio medido con ojos abiertos (OA) y cerrados (OC) sobre una plataforma de fuerza. Se consideraron las variables del centro de presión (CP): área, velocidad media, velocidad mediolateral y velocidad anteroposterior. Se realizó una regresión lineal ajustando por sexo utilizando como variable respuesta las variables del CP y como variable explicativa el sobrepeso/obesidad frente a normopeso.

Resultados: Los niños con sobrepeso/obesidad presentan mayores valores en las variables velocidad media $O A(\beta=0,018 ; p=0,005)$, velocidad mediolateral $O A(\beta=0,122 ; p=0,005)$, velocidad anteroposterior $O A(\beta=0,041 ; p<0,001)$, velocidad media OC $(\beta=0,009 ; p<0,001)$, velocidad mediolateral OC $(\beta=0,067 ; p<0,001)$ y velocidad anteroposterior OC del CP $(\beta=0,409 ; p<0,001)$. Además, se observaron correlaciones significativas entre el aumento del IMC y el deterioro del equilibrio postural $(p<0,05)$.

Conclusiones: Los niños con sobrepeso y obesidad situados entre 6 y 9 años presentan un equilibrio postural más bajo que los normopesos. Con los ojos cerrados, la variable sexo también influye en el control de la postura. 
Influence of nutritional status on postural balance in children: a pilot study

\section{KEYWORDS}

Overweight;

Obesity;

Postural Balance;

Nutritional Status;

Child.

\section{ABSTRACT}

Introduction: Increased body mass index may affect the performance of functional tasks, such as balance, increasing the risk of falls and injuries; however, evidences are limited in children. The aim of this study was to determine the influence of nutritional status on postural balance in children between 6 and 9 years old.

Material and Methods: Cross-sectional descriptive study. 71 children between 6 and 9 years old were evaluated. Weight and height were measured. Balance was measured with eyes-open (EO) and eyes-closed $(E C)$ on a force platform. The following variables were obtained of the center of pressure (CP): area, mean velocity, mediolateral velocity and antero-posterior velocity. Lineal regression was used to explore the association between the CP variables and overweight and obesity versus normal weight adjusted by sex.

Results: The children with excess body mass presented higher values in the CP variables such as mean velocity $E O(\beta=0.018 ; p=0.005)$, mediolateral velocity $E O(\beta=0.122 ; p=0.005)$, anteroposterior velocity EO $(\beta=0.041 ;<0.001)$, mean velocity $E C(\beta=0.009, p<0.001)$, mediolateral velocity $E C$ ( $\beta=$ $0.067, p<0.001)$ and anteroposterior velocity EC $(\beta=0.409 ; p<0.001)$. These last two variables adjusted by sex. In addition, significant correlations were observed between increased BMI and poor postural balance $(p<0.05)$.

Conclusions: Overweight and obese children between 6 and 9 years old have a lower postural balance than normal weight. With eyes closed, the sex variable also influences postural control.

\section{CITA}

Guzmán-Muñoz E, Valdés-Badilla P, Concha-Cisternas Y, Méndez-Rebolledo G, Sazo-Rodríguez S. Influencia del estado nutricional sobre el equilibrio postural en niños: un estudio piloto. Rev Esp Nutr Hum Diet. 2017; 21(1): 49-54. doi: 10.14306/renhyd.21.1.297

\section{INTRODUCCIÓN}

El sobrepeso y la obesidad (S/O) son definidos como una acumulación anormal y excesiva de grasa que puede ser perjudicial para la salud, y que se manifiesta por un exceso de peso y volumen corporal ${ }^{1}$. Se estima que en el mundo $47,1 \%$ de los niños presenta $S / \mathrm{O}^{2}$. En España, las prevalencias de $\mathrm{S} / \mathrm{O}$ en escolares son cercanas al $46 \%$, con mayor presencia en varones ${ }^{3}$. En América Latina, la situación no es más alentadora, siendo Chile una de las regiones más afectadas al mostrar tasas cercanas al $50 \%$ de $\mathrm{S} / 0$ en niños situados entre 5 y 9 años ${ }^{4}$. Este aumento de la obesidad corresponde a un hecho preocupante, debido a que aproximadamente el $70 \%$ de los niños obesos se convierten en adultos obesos ${ }^{5}$.
La clasificación del estado nutricional a partir del índice de masa corporal (IMC), ha demostrado ser un fuerte predictor de mortalidad por enfermedad cardiovascular ${ }^{6}$. Dentro de los principales efectos inmediatos en niños con IMC sobre lo normal destacan alteraciones cardiovasculares, metabólicas, psicológicas y funcionales. Estas últimas, han sido las menos estudiadas ya que su impacto sobre el desempeño en el sistema escolar y la participación social ha sido subestimado. En suma a lo anterior, alteraciones en la coordinación, equilibrio y locomoción podrían afectar negativamente el desarrollo motor del niño, lo que limitaría su participación en actividad física, deportes y tareas de la vida cotidiana? Estas consecuencias funcionales podrían perdurar a largo plazo, independientemente de si su condición nutricional se modifica. En este contexto, resulta de interés estudiar las alteraciones que podría generar el exceso de masa corporal 
Influencia del estado nutricional sobre el equilibrio postural en niños: un estudio piloto

sobre el equilibrio postural, considerando que este es uno de los componentes básicos para desarrollar de manera eficiente gestos motores funcionales ${ }^{8}$.

El equilibrio corresponde a una de las habilidades motoras básicas, fundamental para el desarrollo adecuado de tareas de locomoción como caminar, correr y saltar. Se cree que el aumento de masa corporal puede afectar la realización de estas tareas, lo que eleva la predisposición a lesiones musculoesqueléticas y riesgo de caídas ${ }^{7}$. En este sentido, el método más aceptado para cuantificar el equilibrio postural, corresponde al desplazamiento del centro de presión (CP) por medio de una plataforma de fuerza que mide las oscilaciones posturales ${ }^{9}$. A partir del CP, se pueden obtener registros del área, velocidad media y los componentes mediolateral y anteroposterior de su desplazamiento ${ }^{9}$. Un aumento del valor de estas variables representa una menor capacidad de controlar las oscilaciones posturales, es decir, un peor equilibrio.

Estudios recientes han reportado relación entre el aumento del IMC y el deterioro del equilibrio en adultos ${ }^{10}$. Sin embargo, investigaciones que reporten la influencia del exceso de masa corporal en función del equilibrio postural en niños es limitada. Por lo tanto, el propósito de este estudio es determinar la influencia del estado nutricional sobre el equilibrio postural en niños entre 6 y 9 años de edad.

\section{$\longrightarrow$ \\ MATERIAL Y MÉTODOS}

Se realizó un estudio descriptivo transversal piloto con selección por conveniencia de escolares situados entre primer y cuarto año básico de dos escuelas públicas de la ciudad de Talca, Chile. La muestra incluyó 71 niños entre 6 y 9 años de edad que representa el 30,1\% de la población estudiada. Los requisitos para participar fueron: a) entregar un consentimiento informado firmado por los padres o tutores legales, a quienes previamente se les informó de los objetivos y metodología del estudio; b) asistir a clases el día de las evaluaciones; c) los niños debían ser capaces de comprender instrucciones simples y caminar independientemente. Se consideraron los siguientes criterios de exclusión: a) cirugías de miembros inferiores, b) dolor en cualquier parte del cuerpo al momento de la evaluación, c) trastornos vestibulares, d) trastornos visuales no corregidos y e) uso de ayuda técnica para la deambulación.

Se utilizaron las recomendaciones de la Sociedad Internacional para Avances de la Cineantropometría (ISAK) para las medidas antropométricas ${ }^{11}$. La talla se midió utilizando un estadiómetro (Seca, Hamburgo, Alemania) y el peso corporal se evaluó con una balanza (Seca, Hamburgo, Alemania). El IMC se calculó dividiendo el peso corporal (kg) por la talla al cuadrado $\left(\mathrm{m}^{2}\right)$. Se clasificó a los escolares de acuerdo a su estado nutricional (normopeso, sobrepeso y obeso), en cuanto a las desviaciones estándar de la Organización Mundial de la Salud (normal entre $-1,0$ y +0,9 $\mathrm{DE}$; sobrepeso $+1,0$ a $+1,9 \mathrm{DE}$ y obesidad $\geq+2,0 \mathrm{DE})^{12}$.

El equilibrio se midió sobre un posturógrafo (Artoficio Ltda., Santiago, Chile) con los ojos abiertos (OA) y con los ojos cerrados $(O C)$. Los participantes fueron instruidos en mantener una posición bípeda, lo más quieta posible, durante 30 segundos. En cada situación, se realizaron 3 intentos promediados para la obtención de las variables del CP. A partir del desplazamiento del $C P$, se obtuvieron las siguientes variables: área $\left(\mathrm{m}^{2}\right)$, velocidad media $(\mathrm{m} / \mathrm{s})$, velocidad de dirección medio lateral $(\mathrm{m} / \mathrm{s})$ y velocidad de dirección anteroposterior $(\mathrm{m} / \mathrm{s})$.

Para el análisis estadístico, se utilizó el software SPSS 15.0 (SPSS Inc., IL, EE.UU.). La distribución de normalidad fue comprobada con el test de ShapiroWilk. Se calculó el promedio y desviación estándar para describir las características basales de la muestra (edad, peso y talla). Se utilizó la prueba de correlación de Pearson para identificar la relación entre el IMC y las variables del CP. Un coeficiente de correlación $r$ de 0 a 0,4 se consideró una relación débil, un coeficiente de 0,4 a 0,7 se consideró una relación moderada y un coeficiente de 0,7 a 1,0 se consideró una relación fuerte. Además, se aplicó un modelo de regresión lineal múltiple ajustados por la variable sexo (intervalo de confianza del 95\%) para determinar la influencia del estado nutricional (S/O frente a normopeso) sobre las variables del CP. El nivel de significación para todas las pruebas estadísticas fue de $<0,05$.

\section{RESULTADOS}

La Tabla 1 muestra la media y desviación estándar de las características basales de los participantes clasificados según estado nutricional. En ella se observa que los niños tenían una prevalencia mayor de S/O que las niñas.

En la Tabla 2 se muestra la correlación para el total de la muestra ( $n=71$ ) entre el IMC y los diferentes parámetros obtenidos en el CP, la correlación más alta se observó entre el IMC y la velocidad antero-posterior con los ojos cerrados $(r=0,786 ; p<0,001)$, aunque también se encontraron correlaciones significativas aunque ligeramente inferiores para el resto de variables de $C P$, entre el IMC y la velocidad media ojos abiertos OA ( $r=0,262 ; p=0,013)$, velocidad media de dirección mediolateral con ojos abiertos $(r=0,250 ; p=0,017)$, 
Tabla 1. Características basales de la muestra (media y desviación estándar).

\begin{tabular}{|c|c|c|c|c|c|}
\hline & & Edad (años) & Peso (kg) & Talla (m) & IMC $\left(\mathrm{kg} / \mathrm{m}^{2}\right)$ \\
\hline \multirow{3}{*}{ NORMOPESOS } & Total $(n=19)$ & $7,16(0,74)$ & $25,12(3,35)$ & $1,23(0,05)$ & $16,06(0,67)$ \\
\hline & Hombres $(n=8)$ & $7,13(0,78)$ & $26,38(3,85)$ & $1,26(0,05)$ & $15,93(0,49)$ \\
\hline & Mujeres $(n=11)$ & $7,18(0,72)$ & $24,21(2,58)$ & $1,22(0,04)$ & $16,14(0,77)$ \\
\hline \multirow[t]{3}{*}{ SOBREPESOS } & Total $(n=29)$ & $7,53(0,78)$ & $29,99(3,72)$ & $1,28(0,06)$ & $18,32(0,79)$ \\
\hline & Hombres $(n=16)$ & $7,63(0,69)$ & $29,51(4,06)$ & $1,27(0,07)$ & $18,42(0,78)$ \\
\hline & Mujeres $(n=13)$ & $7,41(0,86)$ & $30,65(3,09)$ & $1,30(0,04)$ & $18,18(0,77)$ \\
\hline \multirow[t]{3}{*}{ OBESOS } & Total $(n=23)$ & $7,04(0,69)$ & $35,58(4,98)$ & $1,27(0,06)$ & $21,76(1,67)$ \\
\hline & Hombres $(n=11)$ & $6,91(0,66)$ & $34,88(6,21)$ & $1,25(0,06)$ & $22,04(2,23)$ \\
\hline & Mujeres $(n=12)$ & $7,23(0,70)$ & $36,86(3,94)$ & $1,30(0,06)$ & $21,67(0,95)$ \\
\hline
\end{tabular}

IMC: índice de masa corporal.

Tabla 2. Resultados de las correlaciones entre el IMC y las variables del centro de presión.

\begin{tabular}{|c|c|c|c|c|c|c|}
\hline & \multicolumn{2}{|c|}{ Total $(n=71)$} & \multicolumn{2}{|c|}{ Hombres $(n=35)$} & \multicolumn{2}{|c|}{ Mujeres $(n=36)$} \\
\hline & $r$ & $\mathrm{p}$ & $\mathbf{r}$ & $\mathbf{p}$ & $r$ & $\mathbf{p}$ \\
\hline \multicolumn{7}{|l|}{ OJOS ABIERTOS } \\
\hline Área $\left(m^{2}\right)$ & 0,060 & 0,615 & 0,111 & 0,263 & 0,156 & 0,179 \\
\hline Velocidad media $(\mathrm{m} / \mathrm{s})$ & 0,262 & $0,013^{*}$ & 0,419 & $0,006^{*}$ & 0,012 & 0,474 \\
\hline Velocidad ML (m/s) & 0,250 & $0,017^{*}$ & 0,304 & $0,031^{*}$ & 0,237 & 0,082 \\
\hline Velocidad AP (m/s) & 0,402 & $<0,001^{*}$ & 0,584 & $<0,001^{*}$ & 0,150 & 0,191 \\
\hline \multicolumn{7}{|l|}{ OJOS CERRADOS } \\
\hline Área $\left(m^{2}\right)$ & 0,137 & 0,127 & 0,108 & 0,214 & 0,411 & 0,006 \\
\hline Velocidad media $(\mathrm{m} / \mathrm{s})$ & 0,552 & $<0,001^{*}$ & 0,607 & $<0,001^{*}$ & 0,071 & 0,339 \\
\hline Velocidad ML (m/s) & 0,674 & $<0,001^{*}$ & 0,630 & $<0,001^{*}$ & 0,598 & $<0,001^{*}$ \\
\hline Velocidad AP (m/s) & 0,786 & $<0,001^{*}$ & 0,789 & $<0,001^{*}$ & 0,587 & $<0,001^{*}$ \\
\hline
\end{tabular}

IMC: índice de masa corporal; CP: centro de presión; AP: anteroposterior; ML: mediolateral;

* Correlación estadísticamente significativa, prueba de Pearson.

velocidad media de dirección anteroposterior con ojos abiertos $(r=0,402 ; p<0,001)$, velocidad media con ojos cerrados $(r=0,552 ; p<0,001)$ y velocidad media de dirección mediolateral con ojos cerrados $(r=0,674 ; p<0,001)$. En hombres ( $n=35)$, la mayor correlación se obtuvo para el IMC y la velocidad media de dirección antero-posterior con los ojos cerrados $(r=0,789 ; p<0,001)$, mientras que en las mujeres $(n=36)$, la mejor correlación se observó para el IMC y la velocidad media de la dirección mediolateral $(r=0,598 ; p<0,001)$ y velocidad media de la dirección anteroposterior $(r=0,587$; $p<0,001)$ ambas con los ojos cerrados.
En la Tabla 3 se muestra la asociación para cada uno de los parámetros de $\mathrm{CP}$ del estado nutricional medido por el IMC como S/O frente a normopeso. El hecho de tener S/O era un factor que se asociaba una mayor puntuación de la velocidad media tanto anteroposterior como mediolateral cuando se realizó el análisis del posturógrafo con los ojos abiertos y cerrados, aunque la mayor asociación se observó para la velocidad media de dirección anteroposterior, en la que ser S/O aumenta en 0,409 (IC95\%:0,275-0,543) puntos la velocidad media comparado con ser normopeso. En la tabla no se muestra la asociación con el área ya que esta no resultó estadísticamente significativa. 
Tabla 3. Modelos de regresión lineal múltiple de las variables del equilibrio postural, ajustados a estado nutricional, sexo y edad.

\begin{tabular}{|c|c|c|c|c|c|}
\hline \multirow{3}{*}{ OJOS ABIERTOS } & \multicolumn{5}{|c|}{ Coeficiente } \\
\hline & \multirow[t]{2}{*}{$\mathbf{R}^{2}$} & \multirow[t]{2}{*}{$\beta^{\text {a }}$} & \multirow[t]{2}{*}{$\mathrm{p}$} & \multicolumn{2}{|c|}{ IC95\% } \\
\hline & & & & & \\
\hline Velocidad media & 0,108 & & & & \\
\hline S/O vs. normopeso & & 0,018 & 0,005 & 0,006 & 0,031 \\
\hline Velocidad ML & 0,107 & & & & \\
\hline S/O vs. normopeso & & 0,122 & 0,005 & 0,037 & 0,206 \\
\hline Velocidad AP & 0,172 & & & & \\
\hline S/O vs. normopeso & & 0,041 & $<0,001$ & 0,019 & 0,063 \\
\hline \multicolumn{6}{|l|}{ OJOS CERRADOS } \\
\hline Velocidad media & 0,266 & & & & \\
\hline S/O vs. normopeso & & 0,009 & $<0,001$ & 0,006 & 0,013 \\
\hline Velocidad ML & 0,369 & & & & \\
\hline S/O vs. normopeso & & 0,067 & $<0,001$ & 0,043 & 0,09 \\
\hline Velocidad AP & 0,498 & & & & \\
\hline S/O vs. normopeso & & 0,409 & $<0,001$ & 0,275 & 0,543 \\
\hline
\end{tabular}

CP: centro de presión; S/O: sobrepeso/obesidad; ML: mediolateral; AP: anteroposterior; a: Coeficiente $\beta$ ajustado por el sexo; IC95\%: intervalo de confianza al 95\%.

\section{DISCUSIÓN}

Los principales resultados de nuestra investigación muestran que los escolares con $\mathrm{S} / \mathrm{O}$ presentan un equilibrio postural deficiente respecto a los normopesos. Un hallazgo complementario de este estudio reportó una fuerte correlación entre el aumento de la velocidad anteroposterior OC y el aumento del IMC $(r=0,786)$. Las variables velocidad anteroposterior $O A(r=0,402)$, velocidad media OC $(r=0,552)$ y velocidad mediolateral $\mathrm{OC}(\mathrm{r}=0,674)$ mostraron una relación moderada con el IMC.

La influencia del exceso de masa corporal sobre el control postural en niños con S/O encontrada coincide con resultados de investigaciones previas ${ }^{13,14}$. Sin embargo, el menor desempeño en la prueba de equilibrio postural observada en los varones no había sido abordado en dicha población. Se ha observado que en edades inferiores a los 11-12 años, las mujeres tienen un mejor equilibrio que los hombres ${ }^{15}$. Se postula que los varones tienen una maduración más tardía del sistema vestibular, lo que afecta directamente el desarrollo del control postura ${ }^{15}$. Esto explicaría la influencia del sexos sobre el equilibrio en la evaluación con $\mathrm{OC}$ y que las correlaciones del presente estudio sean más evidentes en el grupo de hombres.
El exceso de grasa en la región abdominal en las personas con S/O altera la geometría corporal, lo cual genera un desplazamiento anterior del centro de masa. Esto provoca que el sistema motor requiera un mayor torque de tobillo para controlar las oscilaciones posturales en el plano sagital y que el riesgo de caídas aumente cuando los sujetos están expuestos a perturbaciones ${ }^{16}$. Esta hipótesis podría explicar el déficit del equilibrio en dirección anteroposterior observado en los escolares con mayor IMC. Otra explicación se basa en los cambios estructurales que genera el exceso de peso. Los niños con $\mathrm{S} / \mathrm{O}$ tienen pies más planos por el desarrollo de una almohadilla de grasa en el área del medio pie. Este cambio en el apoyo plantar modificaría las zonas de soporte de peso del pie $y$, presuntamente, generaría cambios propioceptivos a nivel plantar que alterarían el mecanismo de control postural, aumentando las oscilaciones posturales en dirección mediolateral ${ }^{17}$. Otros estudios señalan que los cambios antropométricos provocados por el aumento de peso serían el factor principal en la pérdida del equilibrio postural ${ }^{18}$.

Durante $O C$ se observa la influencia del sexo sobre el equilibrio y las correlaciones de mayor fuerza. Cuando se cierran los ojos, existen mayores oscilaciones posturales debido a la inhibición de uno de los sistemas sensoriales que contribuyen al control postural ${ }^{15}$. Por lo tanto, las posibilidades de 
Influencia del estado nutricional sobre el equilibrio postural en niños: un estudio piloto

mantener la estabilidad disminuyen. Esto hace que la prueba con $\mathrm{OC}$ sea más exigente que la prueba con $\mathrm{OA}$ y el déficit del equilibrio sea más evidente.

Dentro de las limitaciones de este estudio se encuentran el pequeño tamaño de la muestra y la selección por conveniencia de los participantes. Esto podría restringir la validez externa del estudio. También, se podría mencionar la utilización del IMC para determinar la influencia del S/O en el equilibrio postural, ya que sólo se limita a valorar la masa corporal y la talla del sujeto. Si bien el IMC ha sido catalogado como un fuerte predictor de mortalidad por enfermedad cardiovascular ${ }^{6}$, algunos estudios señalan que para valorar el efecto del exceso de peso en el equilibrio postural debería considerarse el porcentaje de grasa corporal ${ }^{10}$.

\section{CONCLUSIONES}

Los resultados de esta investigación señalan que los escolares con sobrepeso u obesidad de entre 6 y 9 años de edad presentan un peor equilibrio postural, lo que confirma la influencia del estado nutricional sobre el equilibrio postural en los participantes de este estudio. Se sugiere implementar actividades motoras que incluyan el entrenamiento del control postural en población en etapa escolar, similar a la evaluada en esta investigación.

\section{$\longrightarrow$ \\ CONFLICTO DE INTERESES}

Los autores expresan que no hay conflictos de interés al redactar el manuscrito.

\section{REFERENCIAS}

(1) World Health Organization. Obesity and overweight [Internet]. WHO. [citado 6 de julio de 2016]. Disponible en: http://www. who.int/mediacentre/factsheets/fs311/en/

(2) Ng M, Fleming T, Robinson M, Thomson B, Graetz N, Margono $C$, et al. Global, regional, and national prevalence of overweight and obesity in children and adults during 1980-2013: a systematic analysis for the Global Burden of Disease Study 2013. Lancet. 2014; 384(9945): 766-81.

(3) Sánchez-Cruz J-J, Jiménez-Moleón JJ, Fernández-Quesada F, Sánchez MJ. Prevalence of child and youth obesity in Spain in 2012. Rev Esp Cardiol (Engl Ed). 2013; 66(5): 371-6.
(4) Tobarra E, Castro Ó, Badilla R. Estado nutricional y características socioepidemiológicas de escolares chilenos, OMS 2007. Rev Chil Pediatr. 2015; 86(1): 12-7.

(5) Dehghan M, Akhtar-Danesh N, Merchant AT. Childhood obesity, prevalence and prevention. Nutr J. 2005; 4: 24.

(6) Ortega FB, Sui X, Lavie CJ, Blair SN. Body Mass Index, the Most Widely Used But Also Widely Criticized Index: Would a Criterion Standard Measure of Total Body Fat Be a Better Predictor of Cardiovascular Disease Mortality? Mayo Clin Proc. 2016; 91(4): 443-55.

(7) King AC, Challis JH, Bartok C, Costigan FA, Newell KM. Obesity, mechanical and strength relationships to postural control in adolescence. Gait Posture. 2012; 35(2): 261-5.

(8) Hue O, Simoneau M, Marcotte J, Berrigan F, Doré J, Marceau $P$, et al. Body weight is a strong predictor of postural stability. Gait Posture. 2007; 26(1): 32-8.

(9) Duarte M, Freitas SMSF. Revision of posturography based on force plate for balance evaluation. Rev Bras Fisioter. 2010; 14(3): 183-92.

(10) Meng H, O'Connor DP, Lee B-C, Layne CS, Gorniak SL. Effects of adiposity on postural control and cognition. Gait Posture. 2016; 43: 31-7.

(11) Marfell-Jones MJ, Stewart AD, de Ridder JH. International standards for anthropometric assessment. Lower Hutt, New Zealand: International Society for the Advancement of Kinanthropometry; 2012.

(12) de Onis M, Lobstein T. Defining obesity risk status in the general childhood population: Which cut-offs should we use? Int J Pediatr Obes. 2010; 5(6): 458-60.

(13) Boucher F, Handrigan GA, Mackrous I, Hue O. Childhood obesity affects postural control and aiming performance during an upper limb movement. Gait Posture. 2015; 42(2): 116-21.

(14) Villarrasa-Sapiña I, García-Massó X, Serra-Añó P, GarciaLucerga C, Gonzalez L-M, Lurbe E. Differences in intermittent postural control between normal-weight and obese children. Gait Posture. 2016; 49: 1-6.

(15) Steindl R, Kunz K, Schrott-Fischer A, Scholtz AW. Effect of age and sex on maturation of sensory systems and balance control. Dev Med Child Neurol. 2006; 48(6): 477-82.

(16) Błaszczyk JW, Cieślinska-Swider J, Plewa M, ZahorskaMarkiewicz B, Markiewicz A. Effects of excessive body weight on postural control. J Biomech. 2009; 42(9): 1295-300.

(17) D'Hondt E, Deforche B, De Bourdeaudhuij I, Gentier I, Tanghe A, Shultz $S$, et al. Postural balance under normal and altered sensory conditions in normal-weight and overweight children. Clin Biomech. 2011; 26(1): 84-9.

(18) Gallego AM, Hita F, Lomas-Vega R, Martínez-Amat A. Estudio comparativo del índice de masa corporal y el equilibrio postural en estudiantes universitarios sanos. Fisioterapia. 2011; 33(3): 93-7. 